\title{
POLYCRYSTALLINE MODELLING OF UDIMET 720 FORGING
}

\author{
Julien Thébault ${ }^{1,2}$, Denis Solas ${ }^{2,3}$, Colette Rey ${ }^{1}$, Thierry Baudin ${ }^{3,2}$, Olivier Fandeur ${ }^{4}$, Michel Clavel ${ }^{1}$ \\ 1 MSSMAT, UMR 8579, CNRS ; Ecole Centrale Paris; Châtenay Malabry, 92295, France \\ 2 Univ Paris-Sud, UMR8182, CNRS, ICMMO, Laboratoire de Physico-Chimie de l'Etat Solide ; Orsay, 91405, France \\ 3 CNRS; Orsay, 91405, France \\ 4 CEA Saclay, DEN / DANS / DM2S / SEMT / LM2S; Gif-sur-Yvette, 91191, France
}

Keywords: Deformation, Dynamic Recrystallization, Crystalline Approach, Coupling, Forging

\begin{abstract}
A crystalline modelling of deformation implemented in a finite element code coupled to a recrystallization Cellular Automaton code is proposed and applied to forging processes of superalloys. The coupled modelling is used in order to obtain a better understanding of the microstructural evolution of superalloys during high temperature forging at different strain rates and temperatures. The framework of the modelling is large plastic deformation and large lattice rotation. The used internal variables are dislocations densities on slip systems of the different phases. Modelling is based on viscoplatic constitutive and hardening laws at the scale of the slip systems and describes local strain and stress fields as well as the stored energy and the rotation of the lattice in the grains of the microstructure. At different steps of deformation, formation of subgrains, annihilation of dislocations, nucleation, growth and new orientation of grains are computed.

The 3D aggregates representing the superalloy, are built up from Electron Back Scattered Diffraction method (EBSD) by means of a high resolution Scanning Electron Microscope. The phases are identified by means of EBSD, chemical analysis (EDS) and observations with a Scanning Electron Microscope. In this paper the studied aggregate is realised from a semi product of Udimet 720. Such technique is able to give us, a realistic description of the crystalline orientation, morphology and position of grains in the aggregate. The Finite Element meshing is deduced from the EBSD analysis.

At high temperature, the Udimet 720 is constituted by a $\gamma$ matrix with a Face Centred Cubic structure (FCC) and $\gamma^{\prime}$ precipitates $\left(\mathrm{Ni}_{3}(\mathrm{Ti}, \mathrm{Al})\right)$ with a Simple Cubic structure (SC). The various material parameters used for the coupled modelling are previously determined from compression tests performed at several strain rate and temperature; The dislocation densities are measured from Transmission Electronic Microscope.
\end{abstract}

\section{Introduction}

The Udimet $720^{1}$ alloy is a promising material to build turbine discs. It has been developed to replace classical grades such as Waspaloy and Inconel 718. The strength improvement, obtained by addition of hardening elements, reduces ductility and requires a $\gamma$ ' subsolvus forging. Indeed, for temperatures above the solvus, the $\gamma^{\prime}$ phase is solved causing a strong hardening of the matrix. The grains boundaries behave like weakest links and forging become impossible. In the forging temperature range, the material is composed of two phases with $\gamma$ matrix of FCC structure and an hardening phase $\gamma^{\prime}$ under a precipitate shape $\left(\mathrm{Ni}_{3}(\mathrm{Ti}, \mathrm{Al})\right)$ with a SC structure.

\footnotetext{
${ }^{1}$ Material supplied by Aubert \& Duval who cofinance this study with Ecole Centrale Paris
}

The aim of this study is to predict the microstructural evolution of Udimet 720 during forging at $1100^{\circ} \mathrm{C}$, through a crystalline approach. During high temperature forging, the microstructure is ruled by the simultaneous influence of deformation and dynamic recrystallization. The different mechanisms (plastic deformation, nucleation, grain growth, annealing) depending on temperature, strain rate and deformation ratio respectively, lead to a decrease of the stress (softening) during forging.

In this paper, a crystalline approach using Finite Element method (Cristalecp) is coupled at different steps of the deformation to a recrystallization scheme based on a Cellular Automaton modelling (Reclpces). The used crystalline approach [1,2,3] can describe the interactions between the two phases and can compute the evolution of the local strain and stress fields as well as the density of dislocations and the rotation of the lattice in the different grains. Such crystalline modelling is now well known technique to determine the microstructure effect on the mechanical local behaviour of aggregates $[4,5,6,7,8,9,10,11]$.

Cellular Automaton (CA) and Monte Carlo (MC) algorithms are also standard techniques in computer simulation of static recrystallization processes. The use of such models helps us to understand experimental observations and improves our knowledge of the recrystallization process. In past years, the input microstructure and texture were often assumed to be random [12, 13]. However these assumptions are seldom verified and different procedures were developed to introduce real deformed microstructure $[14,15]$ or simulated deformed microstructure $[16$, $17,18,19]$ as input data in the recrystallization simulation.

The studied aggregate deduced from a semi product by Electron Back Scattered Diffraction method (EBSD), is described in section 2. The two above mentioned approaches are detailed and the material parameters are given in section 3 . The results are presented in section 4. A discussion and a conclusion end this paper.

\section{Material and Aggregate}

The simulated aggregate corresponds to a sample cut in the centre of a semi-product (disc $\varnothing=254 \mathrm{~mm}$ ) supplied by the Aubert\&Duval company. The microstructure aggregate was characterized by Scanning Electron Microscopy (SEM), EBSD and Energy Dispersive Spectroscopy (EDS).

The microstructure of the semi product is given in Figure 1. The matrix ( $\gamma$ phase) is constituted by secondary and tertiary $\gamma^{\prime}$ precipitates $(\varnothing=100 \mathrm{~nm})$ which are not visible at the scale of the Figure 1. These precipitates being solved in the matrix at the 
forging temperature, the matrix is considered as CFC. The grain size of the matrix is the $5-50 \mu \mathrm{m}$ range and many grains present $\Sigma 3$ twin boundaries.

The most part of the primary precipitates $\gamma^{\prime}$ (grain size $1-5 \mu \mathrm{m}$ ) is situated at the grain boundaries. The $\gamma^{\prime}$ structure is SC.

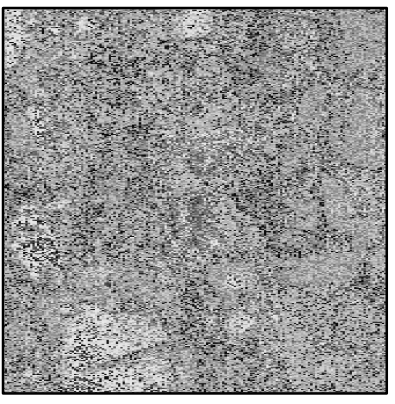

(a) Udimet 720: quality image SEM/EBSD

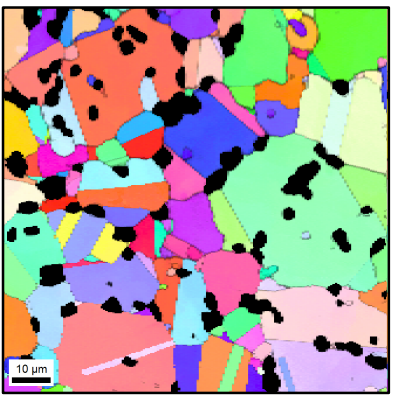
(orientation of the normal to the sample measured by EBSD)

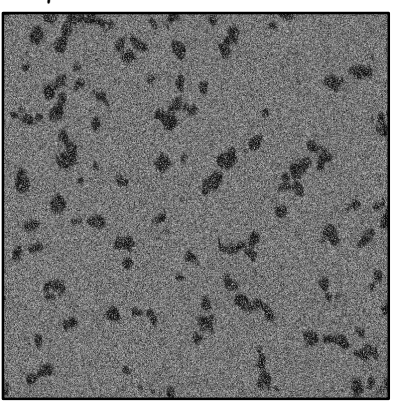

(b) Udimet 720: Precipitates distribution (SEM/EDS)

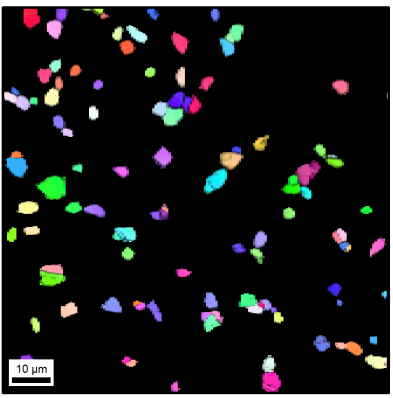

(d) Udimet 720: precipitates $\gamma^{\prime}$ (orientation of the normal to the sample measured by EBSD) (c) Udimet 720: matrix $\gamma$

Figure 1. Microstructural analysis of Udimet 720 by SEM/EBSD/EDS

The material presents a weak texture coming from the different processes before forging. Experimental compression curves, performed at $1100^{\circ} \mathrm{C}$ for different strain rates, point out a large dependence of behaviour with the strain rate (see Figure 2).

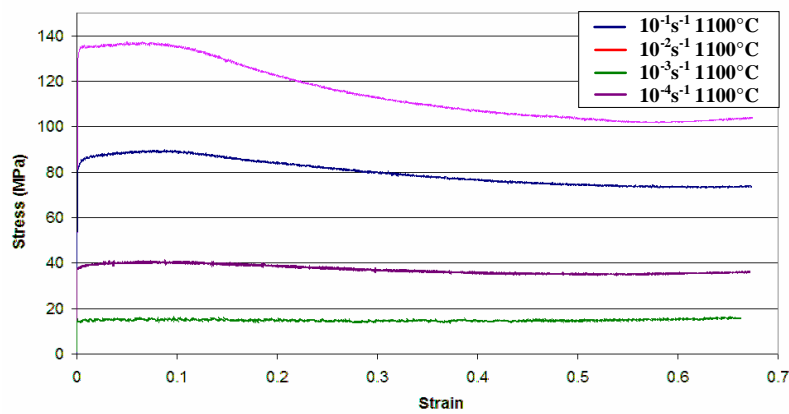

Figure 2. Stress-strain curves for $0.710^{-1} \mathrm{~s}^{-1}, 0.710^{-2} \mathrm{~s}^{-1}, 0.710^{-3} \mathrm{~s}^{-1}$, $0.710^{-4} \mathrm{~s}^{-1}$ respectively

\section{Modelling of the forging}

\section{Crystalline approach}

The Udimet is considered as a two phase material, the matrix and the primary precipitates $\gamma^{\prime}$ are assumed as a FCC and SC structures respectively. The crystalline approach applied for each phase is implemented in the Finite Element Code ABAQUS.

The crystalline plasticity constitutive law uses a large deformation framework, based on the multiplicative composition of the deformation gradient into elastic and plastic parts [4, 20]. For a FCC material, glide occurs on the $12\{111\}<110>$ slip systems and for SC material on the $6\{100\}<110>$ slip systems. The resolved shear stress $\tau^{\mathrm{s}}$, the critical shear stress $\tau_{c}^{s}$ and the slip rate $\dot{\gamma}^{\mathrm{s}}$ are related by a viscoplastic power law:

$$
\begin{aligned}
& \frac{\dot{\gamma}^{s}}{\dot{\gamma}_{0}}=\left|\frac{\tau^{s}}{\tau_{c}^{s}}\right|^{n-1}\left(\frac{\tau^{s}}{\tau_{c}^{s}}\right) \operatorname{sgn}\left(\tau^{s}\right) \text { if }\left|\tau^{s}\right| \geq \tau_{c}^{s} \\
& \dot{\gamma}^{s}=0 \text { otherwise }
\end{aligned}
$$

with $\dot{\gamma}_{0}$ as a reference slip rate. As long as slip occurs, hardening is caused by the interactions between different slip systems. This is usually represented through a phenomenological relationship between a critical shear stress increment and all the slip strain increments weighted with a hardening matrix $h^{\text {su }}$.

$$
\dot{\tau}_{c}^{s}=\sum_{u} h^{s u}\left|\dot{\gamma}^{\mu}\right|
$$

The critical shear stress is also related to the densities of dislocations $\rho^{u}$ on the different glide systems by:

$$
\tau_{c}^{s}=\tau_{0}+\sqrt{\left(\sum_{u} a^{s u} \rho^{u}\right)}
$$

where $\tau_{0}$ contains the friction of the lattice, increased by the effect of alloying elements. $\mathrm{a}^{\text {su }}$ is an interaction matrix which coefficients depend on the interactions between dislocation families (s) and (u).

The hardening matrix expression can be deduced of equations (2), (3) and (4). The hardening matrix $h^{\text {su }}$ evolves with increasing deformation, but depend on internal variables (dislocation densities on all slip systems) in order to take into account the physical aspects of plasticity $[1,2,3]$. On a given slip system $(s)$, the evolution of the dislocation density $\rho^{s}$ is governed by a production term based on Orowan's relationship and by an annihilation term which takes into account the dynamic recovery during deformation. Thus, it may be: 


$$
h^{s u}=\frac{\mu}{2} \frac{a^{s u}}{\sqrt{\sum_{t} a^{s t} \rho^{t}}}\left(\frac{1}{L^{u}}-g_{c} \rho^{u}\right)
$$

where $L^{u}$ represents the mean free path of the mobile dislocations gliding on the system $\mathrm{u}$.

$$
\frac{1}{L^{u}}=\frac{1}{K} \sum_{t \neq u} \rho^{t}+\frac{1}{D}
$$

$\mu$ and $b$ are respectively shear modulus and Burgers vector magnitude; $\mathrm{g}_{\mathrm{c}}$ is proportional to the annihilation distance of dislocation dipoles; $\mathrm{K}$ is a material parameter and $\mathrm{D}$ is the average polycrystal grain size. Only self $\mathrm{a}^{\mathrm{ss}}$ and latent $\mathrm{a}^{\mathrm{su}}$ hardening are considered here. The physical meaning of all the parameters set is detailed in [3].

The parameters used for this simulation were identified using a polycrystalline model and an inverse method from experimental results obtained at different temperatures and deformation rates.

At high temperature and for the two phases, the interaction matrix [21] is assumed weak, which means that dislocations can easily pass through the obstacles due to the latent dislocations.

The material parameters are usually determined by inverse method using results on different mechanical tests. In this case, for high temperature $\left(1100^{\circ} \mathrm{C}\right)$, only experimental compression curves are available, the initial dislocations density in the two phases being determined by TEM. The parameters are presented in Table I.

Table I. Material parameters at $1100^{\circ} \mathrm{C}$

\begin{tabular}{|c|c|c|c|c|c|c|c|c|c|c|c|}
\hline & $\begin{array}{c}\mathbf{E} \\
(\mathbf{G P a})\end{array}$ & $\mathbf{v}$ & $\mathbf{a}^{\text {su }}$ & $\begin{array}{c}\dot{\gamma}_{0} \\
\left(\mathbf{s}^{-\mathbf{1}}\right)\end{array}$ & $\mathbf{n}$ & $\begin{array}{c}\mathbf{g}_{\mathbf{c}} \\
(\mathbf{n m})\end{array}$ & $\mathbf{K}$ & $\begin{array}{c}\boldsymbol{\tau}_{\mathbf{0}} \\
(\mathbf{M P a})\end{array}$ & $\begin{array}{c}\mathbf{\rho}_{\mathbf{0}} \\
\left(\mathbf{m}^{-2}\right)\end{array}$ & $\begin{array}{c}\mathbf{D} \\
(\boldsymbol{\mu m})\end{array}$ & $\begin{array}{c}\mathbf{b} \\
(\mathbf{n m})\end{array}$ \\
\hline $\boldsymbol{\gamma}$ & 126 & 0.3 & $8.10^{-3}$ & $5.10^{-5}$ & 6.2 & 10 & 100 & 5.5 & $10^{14}$ & 10 & 0.26 \\
\hline$\gamma^{\prime}$ & 129 & 0.3 & $8.10^{-5}$ & $10^{-5}$ & 6.2 & 10 & 100 & 5 & $10^{13}$ & 3 & 0.26 \\
\hline
\end{tabular}

The recrystallization model "Reclpces"

After deformation, a recrystallization step is performed. An energy is assigned to each cell as the sum of the stored energy $\mathrm{E}_{\text {stored }}$ and the grain boundary energy $\mathrm{E}_{\mathrm{gb}}$. The stored energy is the result of the Finite Element calculation which gives the local dislocation density. The grain boundary energy depends on the misorientation of a given site with respect to its 6 neighbours [16]:

$$
E_{g b}^{s}=\sum_{s^{\prime}} \gamma^{s s^{\prime}}
$$

where:

$$
\gamma^{s s^{\prime}}=\left\{\begin{array}{l}
\gamma_{m} \frac{\theta^{s s^{\prime}}}{\theta^{*}}\left[1-\ln \left(\frac{\theta^{s s^{\prime}}}{\theta^{*}}\right)\right] \text { when } \theta^{s s^{\prime}} \leq \theta^{*} \\
\gamma_{m} \text { when } \theta^{s s^{\prime}}>\theta^{*}
\end{array}\right.
$$

where $\gamma_{m}$ is the energy of high-angle grain boundaries, $\theta^{*}$ is the misorientation limit for low angle boundaries (taken as $15^{\circ}$ in the present simulations), $\theta^{s s^{\prime}}$ is the crystallographic misorientation between two neighbours sites $s$ and s'. The grain boundary energy as the function of the misorientation corresponds to the well-known Read-Shockley equation. The mobility $\mathrm{M}$ of the boundary between the two sites is calculated according to:

$$
M^{s s^{\prime}}=M_{m}\left[1-\exp \left(-\left(\frac{\theta^{s s^{\prime}}}{10}\right)^{3}\right)\right]
$$

where $\mathrm{M}_{\mathrm{m}}$ is the average mobility of high angle grain boundaries and the misorientation angle is expressed in degrees.

In this simulation, one type of nucleation was implemented: site nucleation with high stored energy, which allows to reproduce the discontinuous dynamic recrystallization mechanism. When the stored energy is larger than a critical value, nucleation occurred. The orientation remains the same and its stored energy is set to zero. Experimentally, it is observed that the nuclei correspond to small volumes which pre-exist in the deformed microstructure.

Concerning the growth step, the reorientation of each site is estimated. For each site, one of its six neighbours is selected at random, this site 2 has to be already in a recrystallized configuration. Then the orientation of site 2 is attributed to site 1 and its stored energy is set to 0 . The variation of the energy $\Delta \mathrm{E}$ between the two configurations is computed. As soon as the driving force is positive the reorientation is not accepted. The velocity $\mathrm{V}$ of the grain boundary is expressed as:

$$
V=-M \Delta E
$$

Where $\mathrm{M}$ is the mobility and $\Delta \mathrm{E}$ is the driving force. During a time increment $t_{i n c}$ the grain boundary moves along a distance $d$. This distance is compared to the cell size $\mathrm{d}_{0}$. The probability of reorientation is expressed as: 


$$
P=\frac{d}{d_{0}}=\frac{-M \Delta E t_{i n c}}{d_{0}}
$$

When all the sites are visited, the microstructure is updated. With this model, a length scale is introduced. As a consequence, using a coherent system of units for the driving force and the grain boundary mobility, a real time scale can be used during the growth step.

\section{Meshing and boundary conditions}

The finite element mesh is constituted by 8 nodes hexahedron with reduced integration (C3D8R) obtained directly from EBSD acquisitions. This analysis has been performed on a semi-product supplied by Aubert \& Duval with a FEG-SEM (LEO 1530) using a $0.6 \mu \mathrm{m}$ step (Figure 3a). Each element of the meshing is characterized by a constitutive law and a dislocation evolution law as well as the initial dislocation density, the initial crystalline orientation and spatial coordinates. By this method, morphology of grains, spatial distribution of phases and crystallographic orientation are taken into consideration, enabling us to describe grains and phases interactions effect on the local mechanical fields.

In order to represent the forging process, boundary conditions of simple compression type are applied on the aggregate.

The sequential steps (deformation-recrystallization) correspond to a deformation of 0.1 . To avoid buckling for these large strains, the pattern thickness is set to $2 \mu \mathrm{m}$.

In this study, the $3 \mathrm{D}$ aggregate is composed of five similar layers and its dimensions are $48.6 \times 48.6 \times 10 \mu \mathrm{m}^{3}$. The purpose is to use five similar layers to study the intermediate layer and so to avoid stresses due to boundary conditions on the edges of the aggregate. The meshing is constituted by 32805 elements.

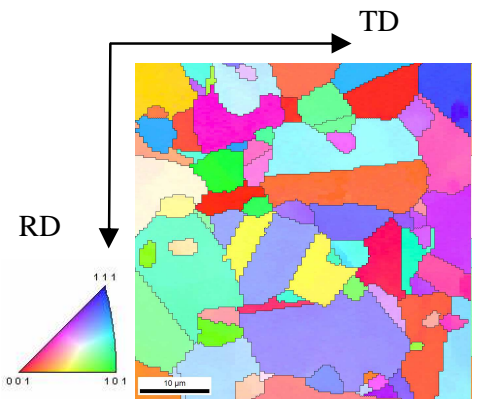

(a)

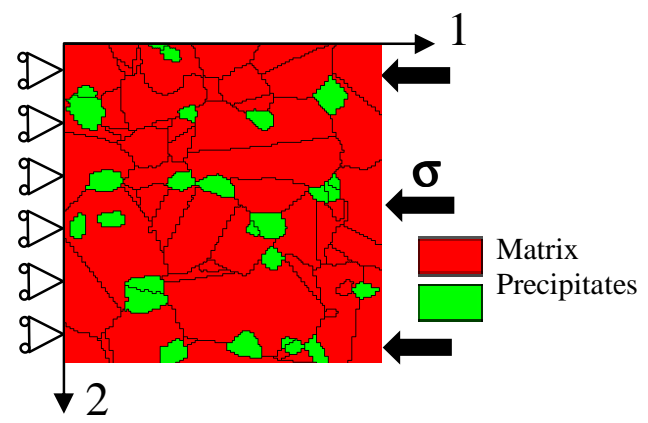

(b)

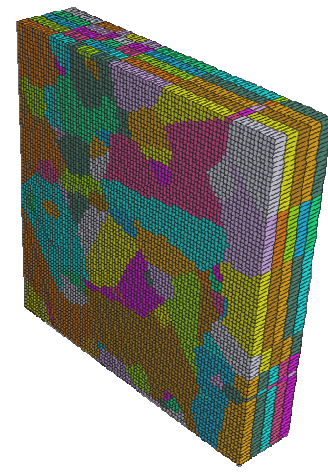

(c)

Figure 3. (a) Example of inverse pole figure map showing the position of the normal axis in the stereographic triangle, (b) phase map, (c) aggregate picture.

\section{$\underline{\text { Sequential coupling }}$}

During high temperature forging, the microstructure evolves under the influence of dynamic recrystallization leading to softening. To perform a sequential coupling of the two modellings, we need to determine the deformation ratio (with "Cristalecp") and the recrystallization time (with "Reclpces"). In this way a third model has been developed. This model corresponds to a macroscopic dynamic recrystallization where deformation part is ensured by a Kocks \& Mecking hardening law (Peczak and Luton 1993) and the recrystallization part is similar to the recrystallization model described above. Figure 4 presents in a theoretical way the different steps of deformation and recrystallization to be achieved in the context of sequential coupling. Recrystallization decreasing stored energy and consequently the macroscopic stress, it is require that the deformation modelling overestimates slightly the results acquired during mechanical tests.

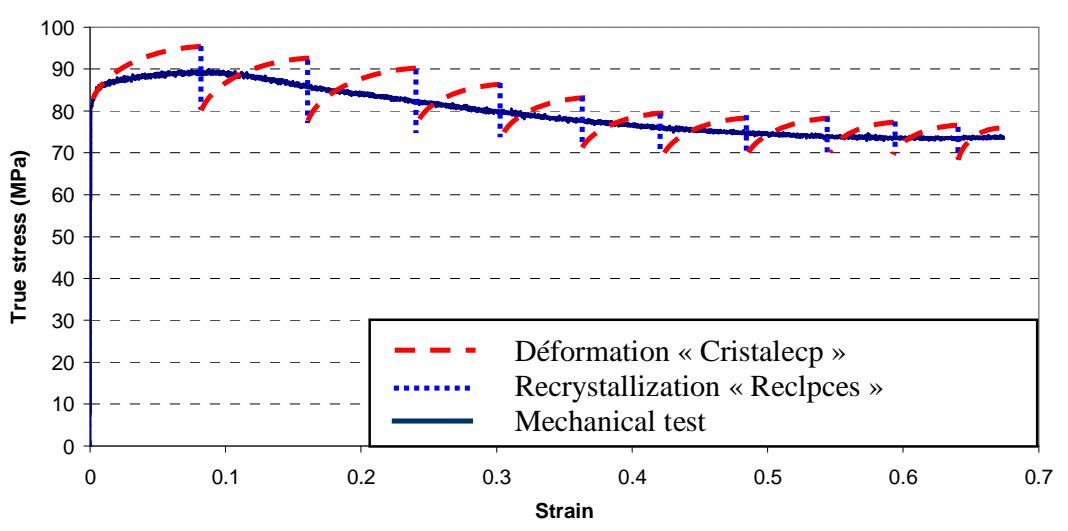

Figure 4. Illustration of sequential approach 
To sum up, sequential coupling requires three models, two for microstructural evolution and one to determine the number and frequency of cycles (deformation + recrystallization).

\section{Results}

This chapter presents the results of the computation obtained after two and a half cycles on the aggregate composed by five similar layers. In order to reduce the computation time, only the intermediate layer is submitted to the sequential coupling.
First deformation: For the first deformation step, it is used parameters shown in Table 1 (named "state 0"). The aggregate is $10 \%$ deformed at a $10^{-2} \mathrm{~s}^{-1}$ strain rate.

To trace stress evolution during sequential coupling, the stressstrain curve is drawn after each deformation step. In the first deformation step, Figure 5 shows that the yield strength is well represented and that the hardening is slightly higher than the experimentally observed one. Thus, during recrystallization, the stress decreases leading to an important microstructural evolution.

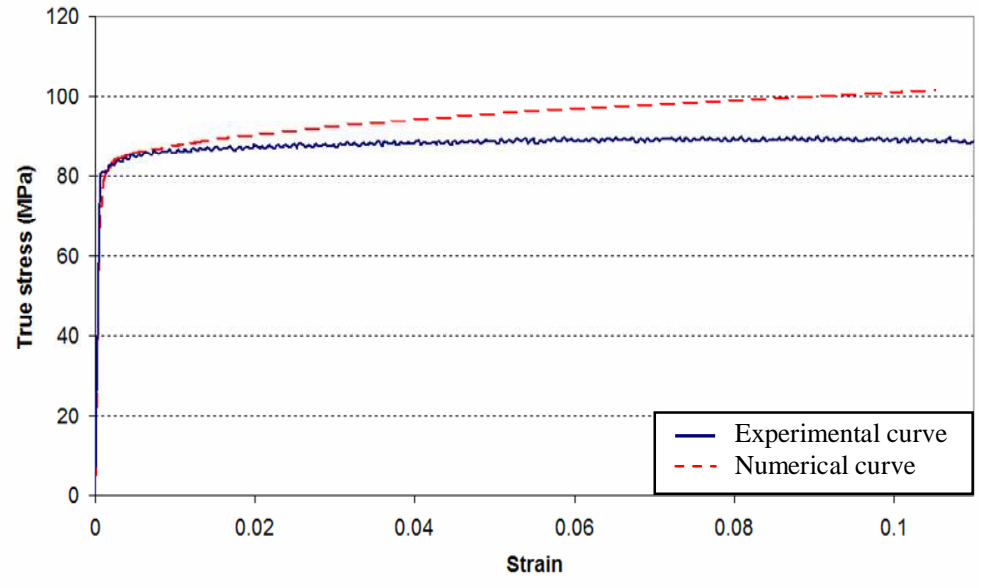

Figure 5. Comparison of simulated and experimental stress-strain curves (first step of deformation)

During deformation, the microstructure evolves by crystal lattice rotation, leading to the formation of intragranular misorientations

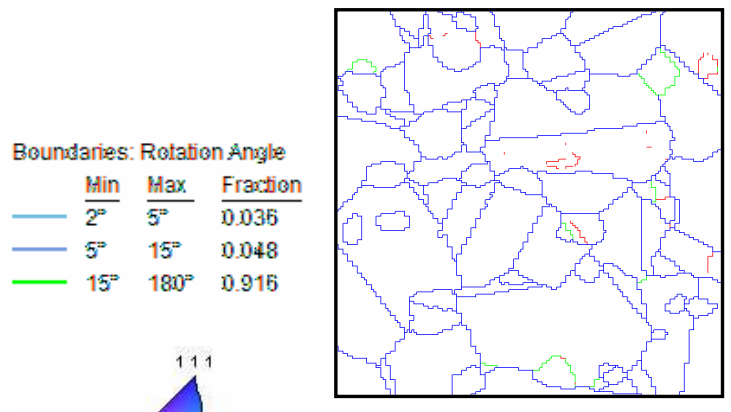

(a)

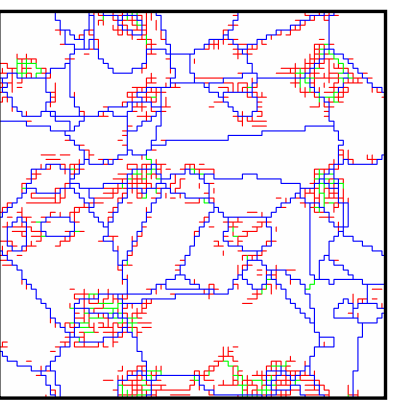

(c) (change of color of grain 1 on Figure 6d) and sub-boundaries (Figure 6c).

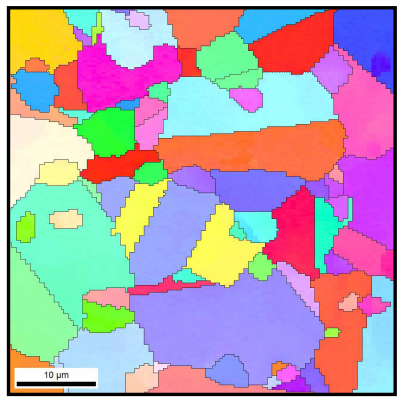

(b)

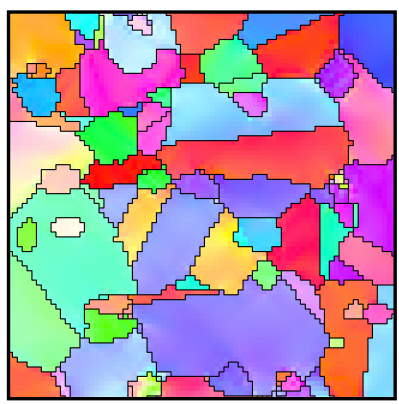

(d)
Initial microstructure $\mathrm{E}=0 \%$

$\mathrm{E}=10 \%$ state 1

Figure 6. Grain boundary map and orientation map before and after deformation 
Recrystallization: After $10 \%$ of deformation, dislocation densities are heterogeneous and nucleation takes place in sites where the energy is higher than a critical value. The comparison of the map at the deformed state (fig7a) and the deformed and recrystallized ones (called state 2) (Figure 7b), points out that some grains have

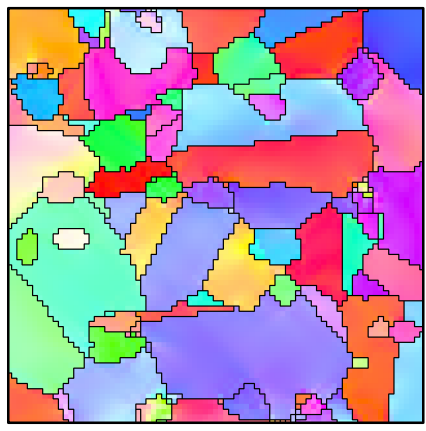

(a)

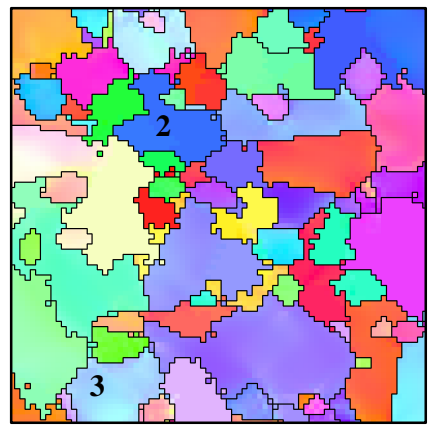

(b) grown and are exempt of dislocations (grain 2) whereas others retain a deformation sub-structure with a crystallographic orientation spread (grain 3) (see Figure 7c). One can also notices that some twin boundaries have vanished.

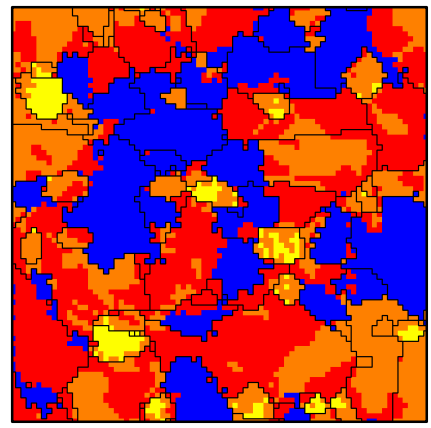

Dislocations densities in $\mathrm{mm}^{-2}$

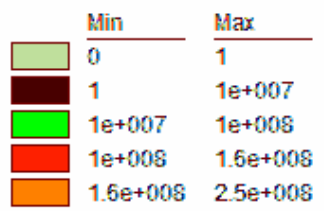

(c)

Figure 7. (a) Orientation map after 10\% of deformation, (b) orientation map after recrystallization,(c) dislocation densities after recrystallization

Second deformation: After the first cycle (deformation + recrystallization), the intermediate recrystallized layer (state2) is inserted in the aggregate at the state 0 (Fig 8.). The role of the surrounding layers is to apply more realistic mechanical fields on the intermediate layer. The intermediate layer differs from the other layers by its heterogeneous dislocation densities and its microstructure. Note that the dislocation densities are calculated in each point from the stored energy after recrystallization.

In the other layers, the dislocation densities are uniform per phase and their values are those of the "state 0".

Initial state : state 0

- Crystallographic orientation given by EBSD

- Unique dislocations density $(\rho)$ per phase

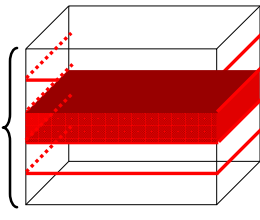

$1^{\text {st }}$ Deformation
Deformed state : state 1

- Crystallographic orientation at state 1

- Increase of $\rho$

- $\rho$ heterogeneous in the aggregate

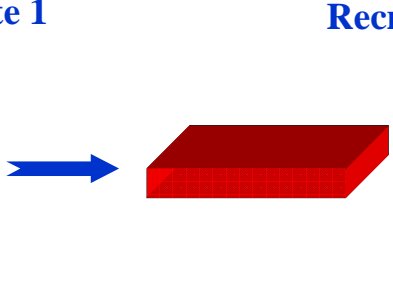

$1^{\text {st }}$ Recrystallization

Recrystallized state : state 2

- Crystallographic orientation at state 2

- $\rho$ heterogeneous in the aggregate

- Recrystallized grains : $\rho=0$

- Deformed Grains : $\rho$ state 1
Input

- Layers at state 0

- Layer at state 2

- Layers at state 0

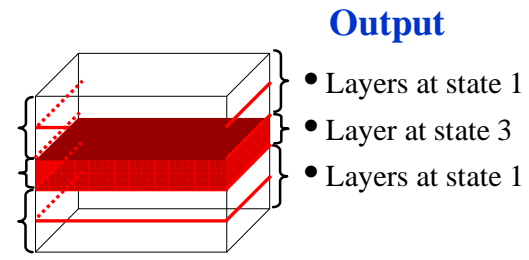

$2^{\text {nd }}$ Deformation

\section{Deformed state : state 3}

- Crystallographic orientation at state 3

- Increase of $\rho$

- $\rho$ heterogeneous in the layer

Figure 8. Inserting a deformed and recrystallized layer into an aggregate at state 0

The aggregate is again submitted to a $10 \%$ deformation with a strain rate of $10^{-2} \mathrm{~s}^{-1}$. After the second deformation $(\mathrm{E}=20 \%)$, the crystallographic orientation of some grains has evolved (grain 4) and the misorientation of some sub-boundaries (located in deformed grains) has increased (Figure 9). 


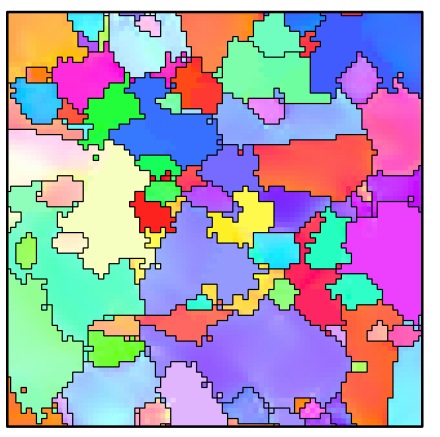

(a)

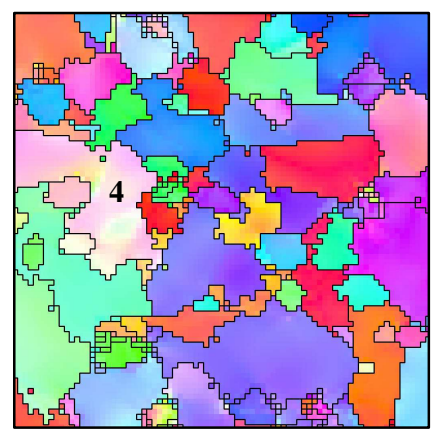

(b)

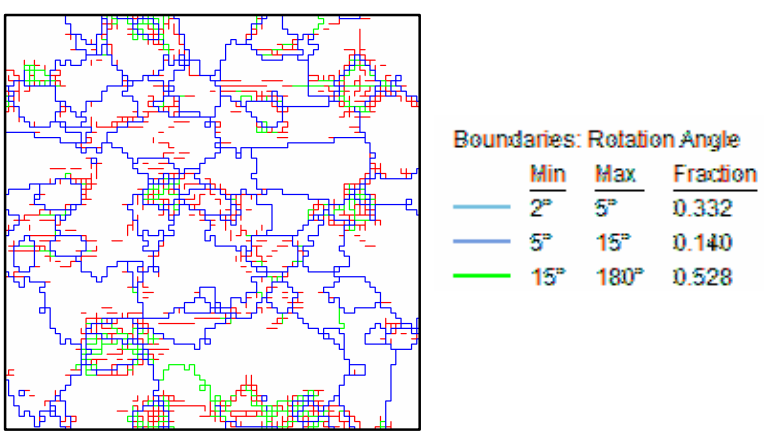

(c)

Figure 9. (a) Orientation map after $E=10 \%$ and recrystallization, (b) orientation map after $20 \%$ of deformation and (c) grain boundary map after the second deformation.

One of the main features of the sequential coupling is to consider, at each step of the computation, all the sub-grains whose misorientation is higher than $2^{\circ}$. Some sub-boundaries created during the first deformation step and not annihilated by the recrystallization can increase their misorientation during the second deformation step.
Microstructural evolution after several cycles: Now the microstructural evolution of the intermediate layer after two and a half cycles is shown on Figure 10.
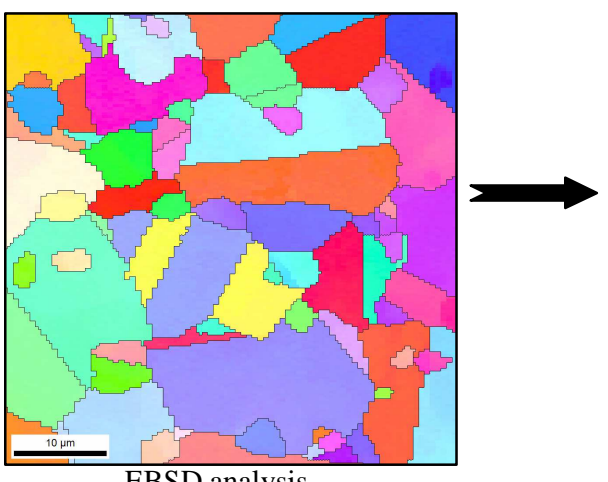

EBSD analysis

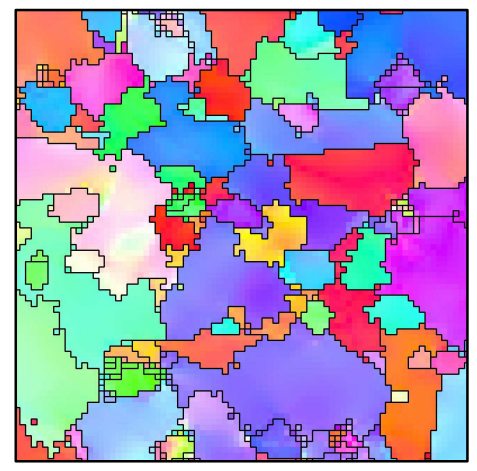

Second deformation

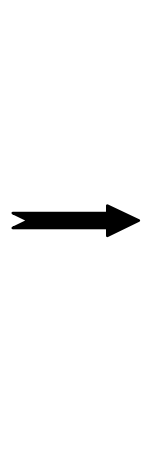

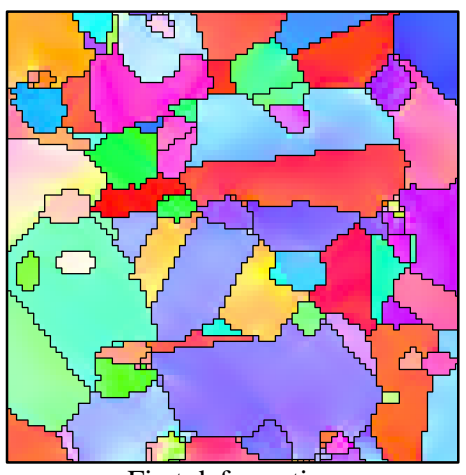

First deformation

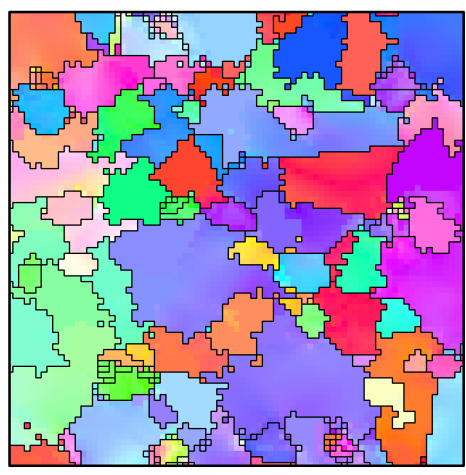

Second recrystallization

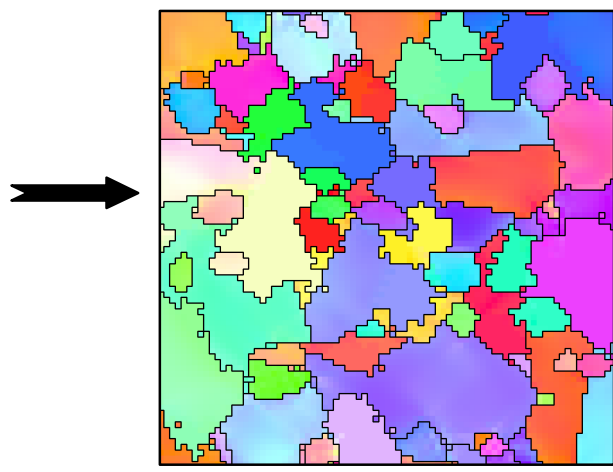

First recrystallization

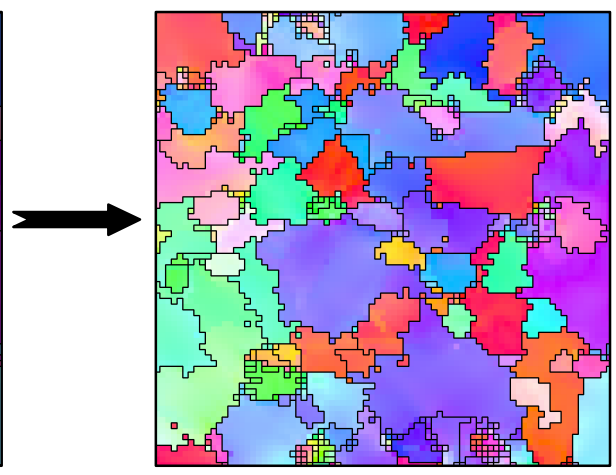

Third deformation

Figure 10. Orientation evolution for two and half cycle of deformation and recrystallization 


\section{Conclusion}

In order to model the high temperature forging of Udimet 720, we developed a procedure of simulation of the microstructures and textures of dynamic recrystallization for a two-phase material. In this way it is used a sequential coupling of models of deformation "Cristalecp" and recrystallization "Reclpces".

Currently the method is operational and the first results are promising. However it still remains to determine more precisely the mechanical parameters for deformation modelling and to take into consideration recrystallization mechanisms identified during experimental analyzes.

\section{References}

[1] T. Hoc, C. Rey, J. Raphanel, « Experimental and numerical analysis of localization during sequentials tests for IF-Ti steel. », Acta metall materiala, 49 (2001), 835-1846.

[2] F. Delaire, J. Raphanel, C. Rey, « Plastic Heterogeneities of a Copper Multicrystal Deformed in Uniaxial Tension : Experimental study and Finite Element Simulations », Acta metall materiala, 48 (2000), 1075-1087.

[3] P. Erieau, C. Rey, « Modelling of deformation and rotation bands and of deformation induced grain boundaries in IF steel aggregate during large plane strain compression.», Inter.J. Plast., 20 (2004), 1763-1788,

[4] D. Peirce, R.J. Asaro and A. Needleman, « Material rate dependence and localized deformation in crystalline solids. ", Acta metall., 31 (1983), 1951-1976.

[5] A.J. Beaudoin, P.R. Dawson, K.K. Mathur and U.F. Kocks, " A hybrid finite element formulation for polycrystal plasticity with consideration of macrostructural and microstructural linking ", Int. J. Plast., 11 (4) (1995), 501-521.

[6] R. Becker and S. Panchanadeeswaran, « Effects of grain interactions on deformation and local texture in polycrystals », Acta metall., 43 (7) (1995), 701-2719.

[7] A. Acharya and A.J. Beaudoin, "Grain-size effect in viscoplastic polycrystals at moderate strains », J. Mech. Phys. Sol., 48 (2000), 2213-2230.

[8] F. Barbe, S. Forest and G. Cailletaud, «Intergranular and intragranular behavior of polycrystalline aggregates.Part 2: Results », Int. J. Plast., 17 (4) (2001), 537-563.

[9] F. Barbe, L. Decker, D. Jeulin and G. Cailletaud, " Intergranular and intragranular behavior of polycrystalline aggregates. Part 1: F.E. model », Int. J. Plast., 17 (4) (2001), 513536.

[10] F.T. Meissonier, E.P. Busso, N.P. O’Dowd, « Finite element implementation of a generalised non-local rate-dependent crystallographic formulation for finite strains », Int. J. Plast., 17 (2001), 601-640.

[11] A. Bhattacharyya, E. El-Danaf, S. Kalindindi and R. Doherty, « Evolution of grain scale microstructure during large strain simple compression of polycrystalline aluminium with quasi columnar grains: OIM measurements and numerical simulations. », Int. J. Plast., 17 (2001), 861-883.

[12] D.J. Srolovitz, G.S. Grest and M.P. Anderson, « Computer simulation of recrystallization-I. Homogeneous nucleation and growth », Acta Metallurgica, 34 (1986), 1863.

[13] D.J Srolovitz, G.S. Grest, M.P. Anderson and R.D. Rollett, « Computer simulation of recrystallization-II. Heterogeneous nucleation and growth », Acta Metallurgica, 36 (1988), 2115.
[14] T. Baudin, P. Paillard and R. Penelle, "Simulation of the anisotropic growth of goss grains in $\mathrm{Fe} 3 \% \mathrm{Si}$ sheets (grade $\mathrm{HiB}$ ) », Scripta Materiala, 40 (1999), 1111.

[15] T. Baudin, F. Julliard, P. Paillard and R. Penelle, " Simulation of primary recrystallization from tem orientation data », Scripta Materiala, 43 (2000), 63-68.

[16] D. E. Solas, C. N. Tomé, O. Engler and H. R. Wenk, «Deformation and recrystallization of hexagonal metals: modeling and experimental results for zinc », Acta Materialia, 49 (18) (2001), 3791-3801.

[17] P. Erieau, « Experimental study and numerical analyzis of the influence of heterogeneities induced by cold deformation on the primary recrystallization of a IF-Ti steel», (Ph.D. thesis, Ecole Centrale de Paris, 2003)

[18] T. Baudin, A.L. Etter, Ph. Gerber, A. Samet, R. Penelle and C. Rey, «Influence of thermo-mechanical treatments on the stored energy simulated by FEM for two low carbon steels », ICOTOM 14, Leuven, Belgium, July 11-15 2005, Trans Tech Publications, Switzerland, Materials Science Forum, 495-497 (2) (2005), 1291-1296.

[19] P. Volovitch, F. Caleyo, T. Baudin, Ph. Gerber, R. Penelle and C. Rey, "Monte Carlo modeling of low carbon steel recrystallization: role of thermo-mechanical treatment and chemical composition », ICOTOM 14, Leuven, Belgium, July 1115 2005, Trans Tech Publications, Switzerland, 1 (2005), 507512 .

[20] C. Teodosiu, J.L. Raphanel et L. Tabourot, « Finite implementation of the large elastoplastic deformation of multicrystals. », Large Plastic Deformation, Proc. Int. Seminar MECAMAT'91, ed.by C. Teodosiu, J.L. Raphanel and F. Sidoroff, (1991), 153-168.

[21] P. Franciosi, "Glide mechanisms in B.C.C. crystals : an investigation of the case of $\alpha$-iron through multislip and latent hardening tests.», Acta metall., 31 (1983), 1331-1342. 